\title{
Intracardiac echocardiography-guided cardiac tumor biopsy: report of the first Slovenian case
}

\author{
(D) Ljupka Dimitrovska*, \\ Bor Antolič, \\ Rok Zbačnik, \\ Matjaž Šinkovec, \\ (D) Jana Ambrožič
}

University Medical Centre Ljubljana, Ljubljana Slovenia

\begin{abstract}
KEYWORDS: imaging, intracardiac echocardiography, endomyocardial biopsy, cardiac tumor. CITATION: Cardiol Croat. 2019;14(3-4):89-90. | https://doi.org/10.15836/ccar2019.89

*ADDRESS FOR CORRESPONDENCE: Ljupka Dimitrovska, Department of Cardiology, University Medical Centre Ljubljana, Zaloška 7, 1000 Ljubljana, Slovenia. / Phone: +386-15224469 / E-mail: ljupkadim@gmail.com ORCID: Ljupka Dimitrovska, https://orcid.org/0000-0002-2467-5438 • Jana Ambrožič, https://orcid.org/0000-0003-4864-7244
\end{abstract}

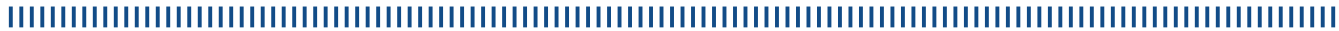

Background: Intracardiac echocardiography (ICE) is an established imaging modality for guiding electrophysiology procedures, with a rapidly expanding role also in other percutaneous structural interventions. Endomyocardial biopsy (EMB) is another potential application where ICE can be used to guide diagnosis of cardiac tumors. ${ }^{1-3}$ Here we present our first case of a cardiac tumor in which EMB was guided by ICE.

Case report: 62-year-old man with a history of non-Hodgkin lymphoma treated with radiation and chemotherapy 10 years ago was admitted due to acute coronary syndrome. Coronary angiogram showed a subtotal occlusion of already collateralized RCA. Due to induced ischemia on myocardial perfusion imaging postponed percutaneous coronary intervention of RCA was performed. During diagnostic work-up at admission when pulmonary embolism was at first suspected, CTA was performed. It showed a heterogeneous formation in the right ventricle (RV) with a thickness of up to $16 \mathrm{~mm}$, that extended from the base towards the apex and into the interventricular septum at the level of the tricuspid ring (Figure 1). According to the CT appearance the mass was suspected to be a liposarcoma Transthoracic echocardiography showed a hypoechogenic form in the RV, clearly defined after application of contrast for left ventricular opacification. Due to previous history of malignancy PET CT was done that revealed hypometabolic RV mass without any sign of distant metastasis. Percutaneous biopsy of the cardiac mass for histopathological diagnosis was performed guided by ICE and fluoroscopy. ICE allowed continuous imaging of intracardiac structures and clear visualization of the cardiac mass in the RV outflow tract under the pulmonary valve (Figure 2). On angiography there was no evidence of flow restriction. A cardiac biopsy catheter was percutaneously inserted into the RV and with the guidance of ICE accurate positioning of the bioptome was achieved. Three tumor specimens were taken for analysis. The histology revealed lipoma, with no evidence of malignancy. In the absence of any cardiac functional disturbances surgical therapy could be avoided and follow-up was proposed.

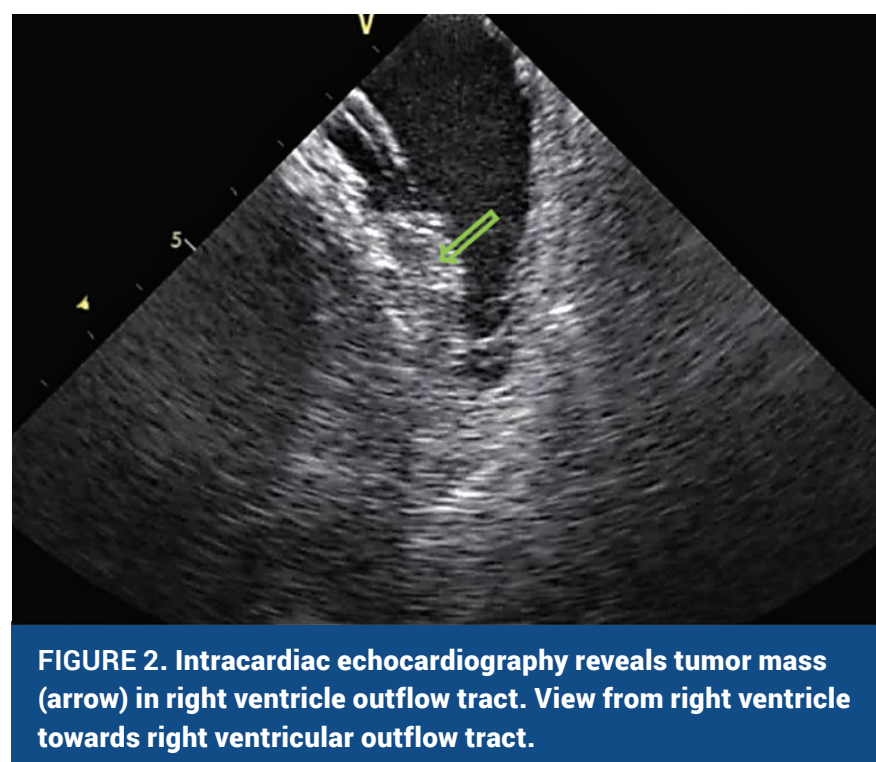


Conclusion: Cardiac masses are rare and often incidental findings. Histology provides definite diagnosis and ICE could be a valuable tool for guiding biopsy procedure. Our first experience confirmed the benefits of ICE that are real-time visualization of cardiac structures, facilitating proper positioning of biopsy catheter, good patient tolerability and the lack of need for general anesthesia. Therefore, with ICE guided percutaneous biopsy the more invasive diagnostic open-heart procedures could be avoided.

1. Kim SS, Hijazi ZM, Lang RM, Knight BP. The use of intracardiac echocardiography and other intracardiac imaging tools to guide noncoronary cardiac interventions. J Am Coll Cardiol. 2009 Jun 9:53(23):2117-28. https://doi.org/10.1016/j.jacc.2009.01.071

2. Bartel T, Müller S, Biviano A, Hahn RT. Why is intracardiac echocardiography helpful? Benefits, costs, and how to learn. Eur Heart J. 2014 Jan;35(2):69-76. https://doi.org/10.1093/eurheartj/eht411

3. Zanobini M, Dello Russo A, Saccocci M, Conti S, De Camilli E, Vettor G, et al. Endomyocardial biopsy quided by intracardiac echocardiography as a key step in intracardiac mass diagnosis. BMC Cardiovasc Disord. 2018 Jan 30;18(1):15. https://doi.org/10.1186/s12872-018-0749-9 